\title{
Detección y tipificación de virus papiloma humano en adenocarcinoma de cuello uterino mediante reverse line blot, Región de La Araucanía, Chile
}

\author{
Priscilla Brebi M ${ }^{1 a}$, Carmen G loria Ili G ${ }^{1 a}$, \\ Jaime López $M^{10}$, Patricia García $M^{1 a}$, Angélica Melo $A^{1 b}$, \\ Sonia Montenegro $H^{1,3 c}$, Pamela Leal $R^{1,4 d}$, \\ Pablo G uzmán G ${ }^{2}$, Juan Carlos Roa $\mathbf{S}^{1,2}$. \\ Detection and genotyping of human \\ papillomavirus in biopsies of uterine \\ cervical adenocarcinoma
}

Background: The genotyping of Human Papillomavirus (HPV) will improve knowledge about the local epidemiological association of this virus with adenocarcinoma. Aim: To determine the frequency of HPV genotypes in biopsies of women with uterine cervical adenocarcinoma in a geographic region of Chile. Materials and Methods: Forty-one cervical biopsies with a pathological diagnosis of adenocarcinoma, corresponding to all women diagnosed with this cancer between 2002 and 2004, were analyzed. Viral gene L1 was amplified by PCR for viral detection. HPV genotyping was carried out by a Reverse Line Blot technique. Results: Seventy one percent of biopsies were positive for HPV. The most common genotypes found were HPV 16 (61\%), followed by HPV 18 (19.5\%). Eighty seven percent of biopsies had a single HPV infection. Three patients had a multiple HPV infection. All of the latter were infected by HPV 16, associated with other three viral genotypes (45, 52 and 66). No low-risk HPV genotypes were found. Conclusions: In this sample of biopsies, there was a bigh prevelence of HPV 16 and a low prevalence of HPV 18, which historically has been related to adenocarcinoma. The genotypes found correspond to those described in South America (Rev Méd Chile 2009; 137: 377-82).

(Key words: Genotype; Human papilloma virus; Uterine cervical neoplasms)

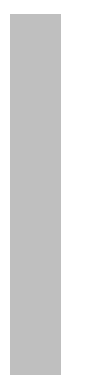

\footnotetext{
Recibido el 24 de junio, 2008. Aceptado el 15 de diciembre, 2008. Trabajo financiado por proyecto DIUFRO DI08-0076 y CORFO INNOVA 07CNI3PBT-222.

${ }^{1}$ Laboratorio de Patología Molecular, Departamento Anatomía Patológica, Facultad de Medicina, Universidad de La Frontera, Temuco, Chile. ${ }^{2}$ Unidad de Anatomía Patológica-Citología, Hospital Dr. Hernán Henríquez Aravena, Temuco, Chile. ${ }^{3}$ Departamento de Especialidades, Facultad de Medicina, Universidad de Concepción, Concepción, Chile.

${ }^{a}$ Estudiante Programa de Doctorado en Ciencias Mención Biología Celular y Molecular Aplicada, Universidad de La Frontera.

bTecnólogo Médico

${ }^{\mathrm{c}}$ Médico Veterinario. Doctor en Biología Molecular

${ }^{\mathrm{d}}$ Ingeniero Agrónomo. Doctor en Biología Celular y Molecular Aplicada
}

\footnotetext{
Correspondencia a: Dr. Juan Carlos Roa S. Departamento de Patología, Facultad de Medicina, Universidad de La Frontera. Manuel Montt 112. Código Postal 478-1176. Temuco, Chile. Fono: 045-325729. Fax: 045-296530.

E mail: jcroa@ufro.cl
} 
$\mathrm{E}^{\mathrm{l}}$ cáncer cervicouterino (CCU) es la segunda neoplasia maligna más frecuente en mujeres y la quinta causa de muerte por cáncer a nivel mundial $^{1}$. Anualmente se diagnostican más de 450.000 casos, con un cuarto de millón de muertes por esta causa ${ }^{2}$. El 80\% de las mujeres afectadas pertenecen a países en vías de desarrollo ${ }^{1}$.

En Chile el CCU ocupa el cuarto lugar dentro de las neoplasias malignas que afectan a la población femenina ${ }^{3}$, siendo la causa de muerte más frecuente entre mujeres de 35 y 54 años ${ }^{4}$. Según datos del Ministerio de Salud en 2005, la incidencia de CCU en Chile es de 18,7 casos por 100.000 mujeres de las cuales fallecen alrededor de 700, siendo la tasa de mortalidad de 8,3 por 100.000 mujeres $^{5}$. En la Región de La Araucanía, constituye la tercera causa de muerte por cáncer, desplazando al cáncer de mama al cuarto lugar ${ }^{3}$.

El CCU puede clasificarse en dos tipos dependiendo del epitelio cervical afectado. El tipo escamoso o epidermoide (SSC) es el más frecuente y se encuentra en 90\% de los casos. El adenocarcinoma (ACC), que afecta al epitelio glandular del cérvix y corresponde aproximadamente a $10 \%$ de los $\mathrm{CCU}^{6}$. Actualmente, la frecuencia del ACC ha ido en aumento desde 5\% hasta 34\% en algunos países ${ }^{7}$. Este hecho se relaciona con la mayor frecuencia de infecciones genitales por virus papiloma humano (HPV), sumado a otros factores como tratamiento anticonceptivo oral y tabaquismo ${ }^{8}$.

El rol del HPV en el desarrollo del CCU se encuentra bien establecido 9,10 , habiéndose acreditado su participación hasta en $99,7 \%$ de los casos $^{8,11}$.

Se han descrito más de 200 tipos virales de HPV de los cuales aproximadamente 40 presentan tropismo por el área anogenital y mucosas, los que pueden ser agrupados en HPV de alto riesgo y bajo riesgo oncogénico dependiendo de su asociación con lesiones de alto grado o carcinomas invasores ${ }^{12}$. Los HPV de bajo riesgo incluyen los tipos 6, 11, 40, 42, 43, 44, 54, 61, 72 y 81 entre los más frecuentes. De ellos los más importantes son los tipos 6 y 11 que producen principalmente condilomas tanto en hombres y mujeres ${ }^{8,13}$. Los HPV de alto riesgo que han sido considerados con potencial oncogénico son los tipos 16, 18, 31, 33, $34,35,39,45,51,52,56,58,59,66,68,70,73$ y 82 por su presencia en lesiones de alto grado y carcinomas. El genotipo más importante y frecuente en el CCU es el HPV 16 $6^{8,13}$.
Se ha observado en algunas regiones geográficas como en Asia-Pacífico, una mayor prevalencia de HPV 18 en ACC, principalmente en mujeres jóvenes $^{14}$. Sin embargo, diversos estudios realizados en Norteamérica, Sudamérica y algunos países de Europa, han demostrado una mayor incidencia de HPV 16. Ambos genotipos son causantes de aproximadamente de $85 \%$ de los casos de $\mathrm{ACC}^{15}$. En Chile son reducidos los datos sobre la epidemiología del HPV y su asociación con el desarrollo de lesiones neoplásicas del cuello uterino, no existiendo publicaciones en el país sobre ACC. Existen diversas metodologías para la detección y tipificación de HPV, entre las cuales destacan Southern blot, hibridación Dot Blot, secuenciación y enzimoinmunoensayo, captura híbrida, reverse line blot (RLB), enzimas de restricción (PCR-RLFP) y PCR en tiempo real ${ }^{16-19}$. RLB es una técnica eficiente, eficaz y económica, siendo capaz de identificar simultáneamente hasta 38 genotipos diferentes. Además es mucho más sensible y específica que las técnicas realizadas mediante enzimas de restricción y dot-blot ${ }^{20}$.

La alta tasa de infección por HPV, principalmente en la adolescencia ${ }^{13}$ y su indiscutible asociación con el desarrollo de CCU llevó a la necesidad de crear vacunas como metodología de prevención primaria. Actualmente, a nivel mundial existen 2 vacunas en el mercado. Ambas incluyen los tipos virales más frecuentes en CCU como son HPV 16 y $18^{21}$. Además estas vacunas presentan reactividad cruzada incompleta con los genotipos virales de alto riesgo oncogénico HPV 31 y $45^{22-24}$.

En consideración con lo expuesto anteriormente se ha propuesto como objetivo determinar la frecuencia de genotipos de HPV en muestras de biopsias de mujeres con diagnóstico de ACC en la región de La Araucanía.

\section{Material y MÉTODO}

Muestras. Se seleccionaron 41 biopsias de cuello uterino (muestras de archivo fijadas en formalina e incluidas en parafina), con diagnóstico histopatológico de adenocarcinoma en la Unidad de Anatomía Patológica - Citología del Hospital Hernán Henríquez Aravena de Temuco, Chile. Cabe mencionar que estas muestras corresponden al total de mujeres con diagnóstico de adenocarcinoma de la región de La Araucanía que consultaron el Policlíni- 
co de Patología Cervical entre los años 2002 y 2004. Este estudio fue autorizado por el Comité de Ética del Servicio de Salud de la Región de La Araucanía.

Extracción ADN. Manualmente, se microdisecaron 8 cortes de 7 micras de cada caso, de acuerdo al área señalada en la placa teñida con hematoxilinaeosina (HE). El tejido fue digerido en buffer de lisis con proteinasa $\mathrm{K}(1 \mathrm{mg} / \mathrm{mL})$. Se precipitó el ADN con isopropanol y finalmente se hidrato para su almacenamiento a $-20^{\circ} \mathrm{C}$ hasta su análisis ${ }^{25}$.

PCR beta globina. Todas las muestras fueron sometidas a un PCR simple para el gen de la beta globina para evaluar la calidad del ADN extraído y excluir así las muestras con $\mathrm{ADN}$ degradado o no amplificable ${ }^{16}$.

Controles para PCR. Como control negativo del PCR específico para HPV se empleó ADN genómico humano (Promega) y como blanco se utilizó mezcla de PCR sin ADN. Como controles positivos para HPV se emplearon plásmidos recombinantes solicitados al ATCC (American Type Culture Collection), probados previamente en diluciones de 100, 50, 10 y 5 pmol/ $\mu \mathrm{L}$, para determinar la sensibilidad del método.

Detección de HPV. Se realizó mediante PCR con los partidores GP5+ y GP6+biotinilado, los que permiten amplificar un fragmento de $150 \mathrm{pb}$ del gen L1 del virus y detectar una amplia gama de tipos de HPV, tanto de alto riesgo $(16,18,26,31,33,35,39,45,51$, $52,53,56,58,59,66,68,70,73$ y 82 subtipos IS39 y W13B/MM4), como de bajo riesgo (6, 11, 34, 40, 42, $43,44,54,55,57,61,71,72,81,83,84$ y CP6108) 20,26 . Tipificación de HPV. Los productos de PCR biotinila- dos fueron sometidos a la técnica de Reverse Line Blot (RLB). Este método permite la hibridación no radiactiva de los productos de PCR biotinilados con diferentes sondas de oligonucleótidos (basadas en lo descrito por Snijders 2006) ${ }^{26,27}$, utilizando un sistema de Miniblotter. Las oligosondas complementarias a cada tipo viral (HPV 6, 11, 16, 18, 26, 31, 33, 34, 35, 39, 40, $42,43,44,45,51,52,53,54,55,56,57,58,59,61,66$, $68,70,71,72,73,81,82$ y 83 , conteniendo un grupo amino en el extremo 5') son unidas en forma covalente a una membrana de nylon cargada negativamente. Después de unir las sondas, la membrana se gira $90^{\circ}$ en el Miniblotter. Las ranuras del Miniblotter, ahora perpendiculares a las líneas de las sondas, son llenadas con productos de PCR marcados con biotina. La hibridación tiene lugar en el Miniblotter y se visualiza usando streptavidina marcada con peroxidasa, que interactúa con el producto de PCR biotinilado, seguido por detección quimioluminiscente mediante autorradiografía. Como control positivo de RLB se utilizó una mezcla de los productos de PCR de plásmidos de HPV. Como control negativo se usó el producto de PCR de ADN genómico y como blanco mezcla de PCR $\sin$ ADN (Figura 1) ${ }^{20,26}$.

\section{Resultados}

La detección del HPV se realizó en 41 muestras individuales con diagnóstico histopatológico de ACC. La edad de las pacientes fluctuó entre 24 y 78 años, con un promedio de 48 años. De las mujeres en estudio, 26,8\% tenían edad entre 24-40 años, $51,2 \%$ entre $41-60$ y $22,0 \%$ sobre 60 años de edad.

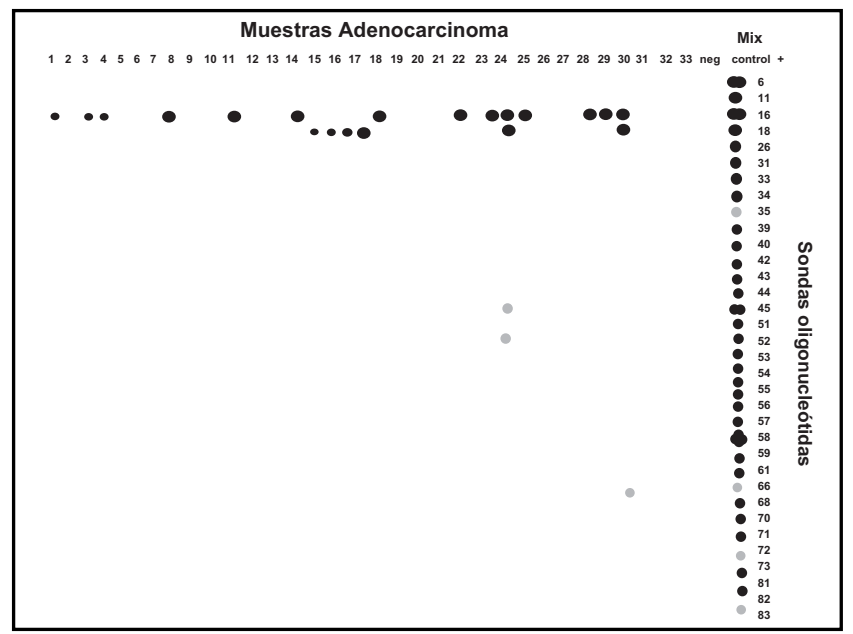

Figura 1. Autorradiografía de la genotipificación de HPV, mediante Reverse line blot. En la figura se observan en forma vertical, los productos de PCR y en forma horizontal las sondas oligonuclótidas complementarias a cada tipo viral. Donde se interceptan las sondas con un tipo viral se genera una hibridación, la cual puede ser puesta en evidencia con Streptavidina marcada con peroxidasa, permitiendo su detección mediante quimioluminiscencia. 
Detección de HPV. Todos los casos estudiados fueron positivos para beta globina, por lo tanto, no hubo exclusión de muestras del estudio debido a calidad del ADN, procediendo posteriormente a realizar PCR del gen viral L1, obteniéndose 29 muestras positivas (70,7\%) y 12 negativas (29,3\%) para HPV (Tabla 1).

Tipificación de HPV. Los genotipos de HPV encontrados en las muestras de ACC fueron: HPV 16 (61,0\%), HPV 18 (19,5\%), HPV 45, 52 у 66 (2,4\% cada uno) (Tabla 1).

Del total de los casos positivos para HPV 86,2\% (61,0\% del total) correspondieron a casos de infección única (IU), es decir, por solo un tipo viral y 13,8\% restante $(9,8 \%$ del total) fueron casos de infección por múltiples tipos virales (IM) (Tabla 1). Los resultados de la tipificación viral en las IU evidencian una frecuencia de HPV 16 de 84,0\%, correspondiendo a más de 50,0\% del total de los casos estudiados, seguido por HPV 18 con 16,0\% de estos casos. Las IM registraron, además de los HPV 16 y 18, tres genotipos virales distintos, los HPV 45, 52 y 66 (Tabla 2).

\section{DisCUSIÓN}

El rol carcinogénico del HPV en el carcinoma escamoso del cuello uterino se encuentra bien descrito en la literatura9,10,12,16, no así su rol en la patogenia del ACC, debido a la baja frecuencia de este tipo dentro de la totalidad de las clases de $\mathrm{CCU}^{7,28}$. En este estudio se analizaron muestras de biopsias de pacientes de la Región de La Araucanía, con ACC de cuello uterino. Entre las edades de 35 y 55 años se obtuvo un mayor número de mujeres infectadas $(56,09 \%)$, lo cual se correlaciona con lo descrito por otros autores ${ }^{4}$. Se confirma una alta asociación entre ACC y HPV que alcanza 70,7\%, coincidiendo con lo reportado por otros autores $7,15,28,29$. Sin embargo, este porcentaje de positividad es levemente inferior pero no estadística-

Tabla 1. Frecuencia de H PV en muestras de biopsia con diagnóstico de adenocarcinoma del cuello uterino

\begin{tabular}{|lccccc|}
\hline $\begin{array}{l}\text { Estatus H PV en } \\
\text { Muestras de ACC }\end{array}$ & $\begin{array}{c}\text { Infecciones } \\
\text { únicas (IU) }\end{array}$ & $\begin{array}{c}\text { Infecciones } \\
\text { múltiples (IM) }\end{array}$ & $\mathbf{n}$ & Porcentaje (\%) \\
\hline HPV negativo & & - & - & 12 & \\
HPV positivo & 25 & 4 & 29 & 70,3 \\
Tipos virales: & HPV 16 & 21 & 4 & 25 & 61,0 \\
& HPV 18 & 4 & 4 & 8 & 19,5 \\
& HPV 45 & 0 & 1 & 1 & 2,4 \\
& HPV 52 & 0 & 1 & 1 & 2,4 \\
Total & HPV 66 & 0 & 1 & 1 & 2,4 \\
\hline
\end{tabular}

ACC: Adenocarcinoma del cuello uterino

Tabla 2. Frecuencia de genotipos de H PV según tipo de infección

\begin{tabular}{|lccr|}
\hline Tipo H PV Infectante & $\mathbf{n}$ & \% sobre IU & \% del total* \\
\hline HPV 16 & 21 & 84 & 51,2 \\
HPV 18 & 4 & 16 & 9,8 \\
\hline Tipo H PV Infectante & $\mathbf{n}$ & \% sobre I M & \% del total* \\
\hline HPV 16/18 & 2 & 50 & 4,9 \\
HPV 16/18/66 & 1 & 25 & 2,4 \\
HPV 16/18/45/52 & 1 & 25 & 2,4 \\
\hline
\end{tabular}

*Porcentaje del total de muestras estudiadas. IU: Infecciones únicas. IM: Infecciones múltiples 
mente significativo a lo observado en otras publicaciones, donde alrededor de $80 \%$ de las muestras presentaban infección por HPV ${ }^{7,29-31}$. Esto se debería a dos posibles causas: primero en el material de archivo, existe una degradación del ADN viral durante los procesos de fijación en formalina, lo que dificulta su amplificación ${ }^{32,33}$ y segundo a que en ACC, debido a que se trata de un epitelio glandular, existe una mayor integración viral, lo que genera un bajo porcentaje de ADN viral en forma episomal, el cual es detectable más eficientemente por técnicas de biología molecu$\operatorname{lar}^{28,32}$.

El HPV 16 ha sido identificado como el genotipo viral más común en carcinoma escamoso del cuello uterino, mientras que HPV 18 se ha relacionado más a ACC y a carcinoma adenoescamoso especialmente en países del Asia-Pacifico ${ }^{18,34,35}$. Sin embargo, otros autores registraron una mayor prevalencia de HPV 16 en casos de ACC tanto en Norteamérica, América Latina y Escocia, respectivamente, coincidiendo con los datos obtenidos en el presente estudio, donde se encontró este genotipo viral en $51,2 \%$ de las infecciones únicas y en todos los casos de infección múltiple ${ }^{31-33}$.

En lugares como África, Asia, Europa y Sudamérica, se menciona una mayor frecuencia de HPV 16, coincidiendo con lo descrito por Trottier, Ferreccio, Muñoz, Clifford entre otros ${ }^{4,13,18,36,37 .}$ Según lo descrito por Muñoz y Bosch en 1999, el genotipo 16 es además el más frecuente en todos los países de Europa, América del Norte, Centroamérica y América del Sur, África y Asia Sudoriental ${ }^{38}$. Esto a excepción de Indonesia, donde el tipo

\section{REFERENCIAS}

1. Longworth MS, Laimins LA. Pathogenesis of human papillomaviruses in differentiating epithelia. Microbiol Mol Biol Rev 2004; 68: 362-72.

2. Parkin DM, Bray FI, Devesa SS. Cancer burden in the year 2000. The global picture. Eur J Cancer 2001; 37 Suppl 8: S4-66.

3. Suárez e, Prieto M, Rojas i, Fernández B, Prado R, Olfos P. Programa nacional de Cáncer Cervicouterino. Rev Chil Obstet Ginecol 2001; 66: 480-91.

4. Ferreccio C, Prado RB, Luzoro AV, Ampuero SL, Snijders PJ, Mejer CJ et al. Population-based prevalence and age distribution of human papillomavirus among women in Santiago, Chile. Cancer Epidemiol Biomarkers Prev 2004; 13: 2271-6.

5. Ministerio de Salud. Guía Clínica Cancer Cervicouterino $21^{\text {st }}$ Ed Santiago: Minsal 2005.
18 es el más común. La distribución de los genotipos virales registrados es similar a lo observado en otros países de Sudamérica como Perú, Brasil, Colombia y Paraguay ${ }^{31}$.

La técnica Reverse Line Blot utilizada para la tipificación de los genotipos virales, presenta ventajas, entre las cuales se destaca que permite discriminar entre HPV de alto y bajo riesgo oncogénico, en forma específica, permitiendo llevar a cabo un estudio de buena calidad experimental ${ }^{20,27}$. En este estudio sólo se registraron HPV de alto riesgo oncogénico. La ausencia de genotipos de bajo riesgo oncogénico incluso en las infecciones múltiples podría indicar una ventaja competitiva de los virus de alto riesgo, prevaleciendo debido a su mejor capacidad de integración genómica en la célula huésped y confirma su alto potencial carcinogénico en esta neoplasia ${ }^{8,12}$. Los genotipos detectados en ACC, son similares a los descritos por otros autores, prevaleciendo HPV 16 y 18 los cuales concuerdan con las altas tasas de frecuencia de ambos tipos virales alrededor del mundo. Asimismo estos resultados indican que la vacunación profiláctica contra la infección por HPV prevendría también el desarrollo de CCU y de la mayoría de los adenocarcinomas en la población chilena ${ }^{30,33}$.

\section{Agradecimientos}

Los autores agradecemos a la Unidad de Anatomía Patológica-Citología, Hospital Dr. Hernán Henríquez Aravena, Temuco por su apoyo en la recolección de muestras. Además, a la Dra. María Alejandra Picconi, por su ayuda en la recopilación de información.

6. Hemminki K, Li X, Vaittinen P. Time trends in the incidence of cervical and other genital squamous cell carcinomas and adenocarcinomas in Sweden, 19581996. Eur J Obstet Gynecol Reprod Biol 2002; 101: 64-9.

7. Castellagague $\mathrm{X}$, Díaz $\mathrm{M}$, de Sanjose $\mathrm{S}$, Munoz $\mathrm{N}$, Herrero R, Franceschi $S$ et al. Worldwide human papillomavirus etiology of cervical adenocarcinoma and its cofactors: implications for screening and prevention. J Natl Cancer Inst 2006; 98: 303-15.

8. Burd EM. Human papillomavirus and cervical cancer. Clin Microbiol Rev 2003; 16: 1-17.

9. Bosch FX, Lorincz A, Munoz N, Mejer CJ, Shah KV. The causal relation between human papillomavirus and cervical cancer. J Clin Pathol 2002; 55: 244-65.

10. Jastreboff AM, Cymet T. Role of the human papilloma virus in the development of cervical intraepithelial neoplasia and malignancy. Postgrad Med J 2002; 78: 225-8. 
11. Motoyama S, Ladines-Llave CA, Luis Villanueva S, Maruo T. The role of human papilloma virus in the molecular biology of cervical carcinogenesis. Kobe J Med Sci 2004; 50: 9-19.

12. Woodman CB, Collins SI, Young LS. The natural history of cervical HPV infection: unresolved issues. Nat Rev Cancer 2007; 7: 11-22.

13. Munoz N, Bosch FX, de Sanjose S, Herrero R, Castellsague X, Shah KV et al. Epidemiologic classification of human papillomavirus types associated with cervical cancer. N Engl J Med 2003; 348: 518-27.

14. Chew G, Cruickshank M, Rooney P, Miller I, Parkin D, Murray G. Human papillomavirus 16 infection in adenocarcinoma of the cervix. BrJ Cancer 2005; 93: 1301-4.

15. Hildesheim A, Berrington de González A. Etiology and prevention of cervical adenocarcinomas. J Natl Cancer Inst 2006; 98: 292-3.

16. Aedo S, Melo A, García P, Guzmán P, Capurro i, Roa JC. Detección y tipificación de virus papilloma humano en lesiones preneoplásicas del cuello uterino mediante PCR-RFLP. Rev Méd Chile 2007; 135: 167-73.

17. Canadas MP, Lloveras B, Lorincz A, Ejarque M, Font R, Bosch FX ET AL. [Assessment of HPV detection assays for use in cervical cancer screening programs]. Salud Publica Mex 2006; 48: 373-8.

18. Clifford GM, Smith JS, Plummer M, Munoz N, FrancesCHI S. Human papillomavirus types in invasive cervical cancer worldwide: a meta-analysis. $\mathrm{Br} J$ Cancer 2003; 88: 63-73.

19. Melo A, Montenegro S, Hooper T, Capurro I, Roa JC, Roa I. [Human papilloma virus (HPV) typing in preneoplastic and neoplastic lesions of the uterine cervix in the IX region-Chile]. Rev Méd Chile 2003; 131: 1382-90.

20. Gravitt PE, Peyton CL, Apple RJ, Wheeler CM. Genotyping of 27 human papillomavirus types by using L1 consensus PCR products by a singlehybridization, reverse line blot detection method. $J$ Clin Microbiol 1998; 36: 3020-7.

21. Markowitz Le, Dunne EF, Saraiya M, Lawson HW, Chesson H, Unger ER. Quadrivalent Human Papillomavirus Vaccine: Recommendations of the Advisory Committee on Immunization Practices (ACIP). MMWR Recomm Rep 2007; 56(RR-2): 1-24.

22. Boot HJ, Wallenburg I, de Melker He, Mangen MJ, Gerritsen AA, van der MaAs NA et al. Assessing the introduction of universal human papillomavirus vaccination for preadolescent girls in The Netherlands. Vaccine 2007; 25: 6245-56.

23. Chan JK, Berek JS. Impact of the human papilloma vaccine on cervical cancer. J Clin Oncol 2007; 25: 2975-82.

24. Mahdavi A, Monk BJ. Vaccines against human papillomavirus and cervical cancer: promises and challenges. Oncologist 2005; 10: 528-38.

25. García P, Benavente F, Melo A, Roa I, Roa J. Effect of fixation on DNA quality: controlled study with five fixatives. Rev Esp Patol 2006; 39: 175-9.
26. van den Brule AJ, Pol R, Fransen-Dahlmeijer N, Schouls LM, Meijer CJ, Snijders PJ. GP5+/6+ PCR followed by reverse line blot analysis enables rapid and highthroughput identification of human papillomavirus genotypes. J Clin Microbiol 2002; 40: 779-87.

27. Snijders PJ, Van den Brule AJ, Jacobs MV, Pol R, Meijer C. HPV DNA Detection and typing in cervical scrapes. In: Davy C, Doorbar J, eds. Methods in Molecular Medicine: Humana Press, 2006.

28. TJalma Wa, Van Waes TR, Van den Eeden LE, Bogers JJ. Role of human papillomavirus in the carcinogenesis of squamous cell carcinoma and adenocarcinoma of the cervix. Best Pract Res Clin Obstet Gynaecol 2005; 19: 469-83.

29. Picconi ma, Alonio LV, Garcia Carranca A, Lizano M, Cervantes Vazquez G, Distefano AL et al. Molecular variants of human papillomavirus (HPV) types 16 and 18 in adenocarcinomas of the cervix. Medicina (B Aires) 2000; 60: 889-94.

30. Peedicayil A, Abraham P, Sathish N, John S, Shah K, SRIDHARAN G ET AL. Human papillomavirus genotypes associated with cervical neoplasia in India. Int $J$ Gynecol Cancer 2006; 16: 1591-5.

31. Santos C, Munoz N, Klug S, Almonte M, Guerrero I, Alvarez M ET AL. HPV types and cofactors causing cervical cancer in Peru. Br J Cancer 2001; 85: 966-71.

32. Pirog EC, Kleter B, Olgac S, Bobkiewicz P, Lindeman J, Quint WG ET AL. Prevalence of human papillomavirus DNA in different histological subtypes of cervical adenocarcinoma. Am J Pathol 2000; 157: 1055-62.

33. Tawfik El-Mansi M, Cuschieri KS, Morris RG, Williams AR. Prevalence of human papillomavirus types 16 and 18 in cervical adenocarcinoma and its precursors in Scottish patients. Int J Gynecol Cancer 2006; 16: 1025-31.

34. Bulk S, Berkhof J, Bulkmans NW, Zielinski GD, Rozendaal L, van Kemenade FJ et al. Preferential risk of HPV16 for squamous cell carcinoma and of HPV18 for adenocarcinoma of the cervix compared to women with normal cytology in The Netherlands. Br J Cancer 2006; 94: 171-5.

35. Iwasawa A, Nieminen P, Lehtinen M, Paavonen J. Human papillomavirus DNA in uterine cervix squamous cell carcinoma and adenocarcinoma detected by polymerase chain reaction. Cancer 1996; 77: 2275-9.

36. Clifford GM, Gallus S, Herrero R, Munoz N, Snijders PJ, Vaccarella S ET al. Worldwide distribution of human papillomavirus types in cytologically normal women in the International Agency for Research on Cancer HPV prevalence surveys: a pooled analysis. Lancet 2005; 366: 991-8.

37. Trottier H, Franco EL. Human papillomavirus and cervical cancer: burden of illness and basis for prevention. Am J Manag Care 2006; 12 (17 Suppl): S462-72.

38. Munoz N, Bosch FX. Relación causal entre virus del papiloma humano y cáncer cervicouterino y consecuencias para la prevención. Bol Oficina Sanit Panam 1999; 121: 550-66. 